\title{
Untangling the relative contribution of maltreatment severity and frequency to type of behavioral outcome in foster youth
}

\author{
Yo Jackson, Ph.D., ABPP, Joy Gabrielli, MA, Kandace Fleming, Ph.D., P. Kalani Makanui, \\ Ph.D., and Angela M. Tunno, MS \\ University of Kansas, Clinical Child Psychology Program, 1000 Sunnyside Ave., Lawrence, KS \\ 66045
}

\begin{abstract}
Within maltreatment research, type, frequency, and severity of abuse are often confounded and not always specifically documented. The result is that samples are often heterogeneous, and the role of components of maltreatment in predicting outcome is unclear. The purpose of the present study was to identify and test the potential unique role of type, frequency, and severity of maltreatment to elucidate each variable's role in predicting outcome behavior. Data from 309 youth in foster care (ages 8-22) and their caregivers were collected using the Modified Maltreatment Classification System and the Behavioral Assessment System for Children, $2^{\text {nd }}$ Edition (BASC2), to measure maltreatment exposure and behavioral outcome respectively. A measurement model of the BASC2 was completed to determine model fit within the sample data. A second confirmatory factor analysis (CFA) was completed to determine the unique contributions of frequency and severity of maltreatment across four types of abuse to externalizing, internalizing, and adaptive behavior. The result of the CFA determined good fit of the BASC2 to the sample data after a few modifications. The result of the second CFA indicated that the paths from severity to externalizing behavior and adaptive behavior (reverse loading) were significant. Paths from frequency of abuse were not predictive of behavioral outcome. Maltreatment is a complex construct and researchers are encouraged to avoid confounding components of abuse that may be differentially related to outcome behavior for youth. Untangling the multifaceted nature of abuse is important and may have implications for identifying specific outcomes for youth exposed to maltreatment.
\end{abstract}

\section{Keywords}

Frequency of abuse; Severity of abuse; Behavioral outcome

Research on mental health outcomes related to maltreatment in youth has developed from perhaps a rather rudimentary and broad categorization of exposure to child abuse to a more

\footnotetext{
(C) 2014 Elsevier Ltd. All rights reserved.

Corresponding author: Yo Jackson, Ph.D., University of Kansas, 1000 Sunnyside Ave., Room 2013, Lawrence, KS 66045 USA, yjackson@ku.edu, 785-864-3581.

Publisher's Disclaimer: This is a PDF file of an unedited manuscript that has been accepted for publication. As a service to our customers we are providing this early version of the manuscript. The manuscript will undergo copyediting, typesetting, and review of the resulting proof before it is published in its final citable form. Please note that during the production process errors may be discovered which could affect the content, and all legal disclaimers that apply to the journal pertain.
} 
fluid and meaningful construct reflecting the multitudes of ways youth are impacted by trauma. A variety of both short- and long-term outcomes potentially related to maltreatment have been tested ranging from externalizing to internalizing behaviors to dissociative symptoms and self-harm behaviors to adaptability and coping skills (i.e., Bryant \& Range, 1997; Lau \& Weisz, 2003; Thompson \& Tabone, 2010). The result of this work thus far documents a significant relation between exposure to maltreatment and a wide range of outcomes that suggest exposure to child maltreatment is a non-specific risk factor associated with many negative outcomes (Afifi, McMillen, Asmundson, Pietrizak, \& Sareen, 2011).

Although few doubt the risk that exposure to maltreatment poses, findings that indicate child maltreatment is associated with such varied outcomes suggests that the field may have yet to provide a clear and useful way to operationalize the maltreatment construct in research.

Thus, although it is important for research to continue to explore how maltreatment relates to mental health, an important first step may have been overlooked; namely, how maltreatment is defined and quantified. Understanding that as the knowledge base grows, so grows the sophistication of the field's approach to research questions, recently, the field has begun to appreciate the meaningful differences in how maltreatment is conceptualized in research. In a special issue of Child Abuse and Neglect in 2005, several researchers attempted to articulate empirically the complexity of the child maltreatment variable and examine how severity, type, and frequency of maltreatment may be differentially related to child outcomes. For example, Litrownik et al. (2005) found that severity could be conceptualized as the maximum severity within or across types of abuse with maximum severity by type related to externalizing behavior and mean severity by type predictive of adaptive behavior. English et al. (2005) found that type of abuse (and severity) was an important consideration and that when examined together (i.e., physical abuse, sexual abuse, and neglect) different types of abuse predicted different mental health outcomes (e.g., physical abuse and neglect and not sexual abuse predicted socialization skills).

Results from studies such as these are important and begin to point the way to a finer grained analysis of what is meant by the finding that maltreatment predicts maladjustment. Despite finding that type of abuse, severity of exposure, and frequency of occurrence are important and often meaningfully related distinctions in predicting mental health outcome, the majority of research continues to examine these features in isolation or in some inconsistent combination (Fassler, Amodeo, Griffin, Clay, \& Ellis, 2005). Drawing upon past research (i.e., Lau et al., 2005) the present study sought to extend the notion that the way that maltreatment is defined matters in the prediction of outcome behavior by examining type, severity, and frequency together. Rarely has the multidimensional nature of maltreatment been tested concurrently or its dimensions examined for their individual roles in the robust maltreatment-maladjustment relation. The present study examined the unique contribution of the most commonly identified dimensions of maltreatment to determine how each component may be differentially predictive of mental health outcomes in youth.

\section{Function and Utility of Definitions of Maltreatment in Research}

Operationally, measurement of maltreatment has moved from primarily retrospective reports from adults of events from childhood (Higgins \& McCabe, 2001) to more real-time 
assessment of youth currently or recently involved in the foster care system (Pears, Kim, \& Fisher, 2008). A review of the research on child maltreatment over the past 10 years suggests that the aim of most studies in youth is to determine how having a history of maltreatment is related to some other variable (Tanaka, Werkerle, Schmuck, \& Paglia-Boak, 2011).

Clearly, this work is important and the robust findings across numerous studies of maltreatment show significant and meaningful differences between children who do and do not have a history of maltreatment (i.e., Lenneke, Cicchetti, Jungmeen, \& Rogosch, 2012). What is less clear is what significant group differences mean. That is, exposure to child maltreatment is not a unitary construct where when present for one child, necessarily means the same thing when present for another (Keiley, Howe, Dodge, Bates, \& Petit, 2001). Although researchers often document the kind or number of abuse events in their groups of maltreated youth (e.g., at least one exposure to physical abuse, youth with a history of sexual abuse), within group differences are rarely examined prior to analyses testing group membership and outcome differences. Within group differences among youth exposed to maltreatment matter, however, as even preliminary investigations show that within the same sample, the timing of certain kinds of abuse at different ages and at different levels of severity can predict different outcomes (English et al., 2005). Although the components like type and severity of abuse may be naturally overlapping, by failing to account for this overlap, it is not clear if it was the physical component or the severity component or both that were the mechanism accounting for maladjustment.

Moreover, in addition to problems with the operationalization of maltreatment, prior work has used varied approaches to data collection methodology. Some research relies not on selfreport, but on case file report or substantiation of abuse as the indicator of maltreatment history group membership (Currie \& Spatz Widom, 2010; Mills et al., 2011). If comparison of results between studies is the goal to building a database of evidence, readers of the field would have to take some caution in considering if the available data represents a consensus or accurate picture. Substantiation or documentation of abuse suggests an entirely different level of verification for group membership than self-report, as some types of abuse are simply harder to substantiate than others (Slep \& Heyman, 2006). Further, when abuse can be documented or substantiated, it often means the abuse event is likely, no matter the type, a severe event where either physical or medical indicators are evident (Fantuzzo, Perlman, \& Dobbins, 2010).

Whether case file report or self-report, how much abuse and how damaging the event was are all common considerations of research within the field of child maltreatment. Each component is important, but because these components are often intertwined (e.g., comparing severity of sexual abuse with severity of physical abuse); it is hard to know if it is the type or the degree of threat posed by the event (i.e., severity) that accounts for its relation to outcomes. The present study sought to attempt to untangle abuse components and to clarify the role of type, frequency, and severity and their relation to behavioral outcomes. 


\section{Type of Abuse}

Research has documented the numerous ways that children are identified as maltreated with the earliest efforts focused on identifying different types of maltreatment (Rutter, 1997). The field appears to have agreed (as evidenced by commonly used research methodology) that maltreatment is composed of at least four distinct types of abuse: physical, sexual, emotional, and neglect (Leeb, Paluzzi, Melanson, Simon, \& Arias, 2008). Moreover, some research has indicated that specific types of abuse are associated with more or less of a particular type of maladjustment (Lobbetael, Arntz, \& Bernstein, 2010; Minrova et al., 2011). For example, in a review of the literature, Maniglio (2010) found that sexual abuse was a risk factor for depression across studies utilizing both youth and adult samples. The effect ranged from small-to-moderate and was likely moderated by other traumatic experiences.

Although a compelling idea that a specific cause has a specific effect, most of the research that attempts to document a direct relation between maltreatment and outcomes is hampered by the notion that abuse types do not necessarily occur in isolation. That is, many youth exposed to abuse are often exposed to more than one kind of abuse, and when youth who are maltreated are examined for the predictive power for a given type of abuse to produce a given kind of outcome, most research fails to account for the overlap with other types of abuse experienced either by omission (failing to measure the possible overlap) or commission (by ignoring the overlap when measured for the sake of clarity; Mendle et al., 2010). Although cross-study comparisons are hampered by the use of varied measures for determining and documenting data measuring types, a review by Herrenkohl and Herrenkohl (2009) echoes the findings of other authors (i.e., Higgins \& McCabe, 2001), namely that a significant portion of youth experience multiple types of abuse.

Methodological limitations aside and as theoretically attractive as it may be to consider types of abuse as independent variables, the data in research studies does not always bear out the notion that types of abuse are actually different constructs (Arata et al., 2005; Kinard, 2001). That is, data shows that types of abuse are often significantly correlated, which suggest that at the least, the tools used to capture type may possess some confounds. Emotional abuse and physical abuse are correlated as high as .78 (Higgins \& McCabe, 2003), physical abuse and neglect correlated as high as .63 (McGee, Wolfe, Yuen, Wilson, \& Carnochan, 1995), physical and sexual as highly as .51, and emotional abuse and neglect at .74 (Higgins \& McCabe, 2003). Herrenkohl and Herrenkohl (2009) concluded that one method, specifically self-report, tends to have the highest correlation among abuse types and the lowest correlations are found when case file report is used. It is likely that because case file report is most often limited to documenting only one reason per hotline call or one reason for admission to foster care, only one type of abuse is documented.

Finally, when research does consider multiple types of abuse present in one individual history, the data is beginning to demonstrate support for the cumulative risk model suggested by Jaffee and colleagues (2007), specifically, that other stressors, or being exposed to more than one abuse type, may also be important to consider in interpreting the meaning of the abuse-outcome relation. For example, Hahm, Lee, Ozonoff, and Wert (2010) 
found that youth experiencing sexual abuse plus other maltreatment types had poorer outcomes compared to individuals who reported only one type or multiple non-sexual abuse events. Although a good start in understanding the importance of considering multiple types, studies like Hahm and colleagues that do not account for how often the abuse occurred may risk representing a partial but incomplete picture of the maltreatment-outcome relation.

\section{Frequency}

Despite the notion that how much or how frequently one experiences abuse could be important for how one is affected by abuse, the notion of frequency in maltreatment research is usually examined only as a broad indicator of rate of reoccurrence or re-victimization (DePanfils, \& Zurvain, 1998). Perhaps the potentially obtuse measurement of frequency is understandable given that research on the number of times a child is abused is a challenge, especially if the data for inclusion is based on case file report or retrospective memory for events in childhood. For the former, it is likely that the maltreatment that comes to the attention of authorities does not represent all of the maltreatment the child has experienced in the past, and for the latter, memory for events may not be accurate (Loftus, 2005). Moreover, in any study, abuse is usually measured as a present or absent variable, something that did or did not happen; little attention is paid to how many times one was neglected or injured (Ahmadkhaniha, Shariat, Torkaman-Nejad, \& Moghadam, 2007). Research on other traumatic events outside of maltreatment research, however, seems to appreciate that dose matters, as studies on chronic medical conditions or environmental conditions like community violence or poverty clearly indicate that the number of times a child is exposed to threat is important for both pathology and adaptive reactions (Miller, Chen, \& Parker, 2011).

One possible exception in the maltreatment literature is a recent study in Canada where chronicity of maltreatment was documented over the course of six years, and youth were grouped into chronic and transitory maltreatment groups based on if they continued to have an open child protective services file over the course of the study (Ethier, Lemelin, \& Lasharite, 2004). The results indicated that children in the chronic maltreatment group had more psychological problems (i.e., anxiety, aggression) and demonstrated higher or clinical levels of symptoms compared to youth whose abuse history was documented by authorities as shorter-term. The Ethier et al. study is also a good example of the difficulty in the conceptualization of frequency of maltreatment, that is, often number of times an event occurred is actually captured in the literature as length of time. Chronicity can be conflated with frequency, the idea that abuse is persistent and stable and it is this stability and not frequent or repeated abuse events that is relevant to outcomes. Furthermore, given that allegations of neglect are sometimes in and of themselves insufficient cause for child removal, it is often the case that length of time confounds with neglect experiences and further complicates the quantification of the "amount" of neglect a child has ultimately experienced.

One could make the claim that the good and important findings from research on chronicity (Bolger \& Patterson, 2001) do not really address the frequency or dose of abuse question. For example, Manly et al. (1994) examined the link between chronicity and behavioral 
outcomes by defining chronicity as the number of months a child had an open case with child protective services. Although this study did not capture the number of times the child was abused, the findings did show that chronicity, over and above the influence of maltreatment, was related to aggressive behavior.

Several longitudinal studies also support the importance of chronicity, as indicated by length of time. For example, Bolger and Patterson (2001) found that youth were rated as more aggressive and at greater risk to be rejected by their peers if their maltreatment was chronic (case file documenting abuse over several years) compared to youth whose case file was open relatively briefly. Although for some types of abuse, such as neglect, the span of time that one is exposed to maltreatment may make conceptual sense (e.g., how long one is left unsupervised or lives in an unsafe home) for chronicity to be measured as length of time a case file is open. For physical and sexual abuse, however, this approach may be less meaningful. That is, physical and sexual abuse are generally acute events, and what may really be important is the number of times one is assaulted, even if those assaults take place over a fairly short period of time or were quite brief in duration. Moreover, there are many reasons why a child's case file might stay open with child protective services, and only one of those reasons is because of ongoing or chronic abuse (e.g., state mandates supervision of family well-being for a period of time).

Finally, studies of chronicity or frequency have as their focus the repetition (either amount over time or total number of events) of abuse and it is likely that multiple or prolonged events have a different impact on youth depending on how severe each event was; it is possible that what really matters is how damaging a given single event was, even when the child may have a long history of multiple low-impact maltreatment exposures. To better identify the role of frequency and severity, the present study addressed each of these variables as separate constructs to determine the relative contribution of each to behavioral outcome.

\section{Severity}

Of the three components of abuse experience often explored in the literature, severity of abuse is perhaps the one with the most agreement among researchers. Data from the field consistently demonstrates that events that are more invasive, or that leave lasting disability or injury to youth, are considered more severe than those whose physical effect is timelimited (e.g., acts that result in physical scars or impaired physical functioning compared to acts that result in minor cuts or bruises). Several complex and useful models and methods exist to assist researchers in documenting the level of injury or damage of a given abuse event. For example, the Maltreatment Classification System (Barnett et al., 1993), which was modified by the LONGSCAN investigators (2005), is widely used in the field. Runyan and Litrownik (2003) found 80-90\% agreement between the severity codes provided by case file data and the codes of severity when using the Modified Maltreatment Classification System (MMCS). So far, the utility of the MMCS has been restricted to documenting severity in case file data only, but could be a useful tool for documenting severity of abuse in self-report. 
Regardless of the reporter, however, the data thus far suggest that the more severe a child's exposure (e.g., the more invasive, the more physically debilitating), the greater the negative impact on a child's well-being and development of adaptive skills (Bryant \& Range, 1997). For example, Bryant and Range found that more severe experiences of physical abuse and sexual abuse were related to more significant problems of suicidality, fewer reasons for living, and fewer coping beliefs suggesting that severity of maltreatment was related to both maladaptive and adaptive outcomes.

Although the construct of severity may appear to be straightforward at first glance, researchers also have to contend with the possible confound of abuse type. That is, for some types, namely physical abuse, the severity indicator (i.e., lasting physical damage to the child) may be easier to document than for other types, such as sexual abuse or neglect (Trocme et al., 2003). Because type and severity can be confounded, it is unclear what the unique contribution is to the maltreatment-outcome relation when the maltreatment variable is a mix of type and severity. The overlap between the components of maltreatment (i.e., type, frequency, and severity) makes documenting the role of maltreatment on adjustment challenging. Moreover, because most research does not consider the possible confounds within the measurement of maltreatment, it can be hard to compare the results of studies on abuse or to understand how one study informs the results of another.

\section{Limits of past research and current study}

Although it is likely the grand consensus in the field that maltreatment in youth is related to maladjustment and adaptability, how maltreatment is conceptualized matters. The way that the field had arrived at that conclusion was through a myriad of studies, defining the presence of maltreatment as sometimes the kinds or types of experiences, the frequency of exposures, the severity of events, or in some cases, a combination of all three. Because the experience of maltreatment is not always acute and can range from one or two severe events to multiple different types of events in the same person's life history, the lack of consistent measurement of the construct may have resulted in some confusion for the field. That is, it is not clear how best to conceptualize the construct of maltreatment in research so that previous relations with maladjustment can be clear and predictable.

Because most youth who are maltreated are exposed to more than one type of abuse, and because not all maltreatment experiences are the same, the present study sought to add to the field by empirically testing the most commonly identified components of the construct of maltreatment to determine their unique contribution to mental health outcomes. With type of abuse included it was predicted that frequency and severity could be modeled as unique constructs with unique variance significantly predictive of behavioral outcomes.

\section{Method}

\section{Participants}

Eligible participants were 309 children in the legal custody of the Division of Family Services between the ages of 8 and 22 years with a mean age of 13.28 years $(S D=3.04)$ sampled from a large, Midwestern city in the United States. All children were in foster care 
with about half in residential facilities (53\%) and the remaining youth in traditional homebased settings. Almost half of the youth participants were female ( $43 \%$ female). Adult caregivers providing reports on the emotional and behavioral functioning of children were predominantly staff or caseworkers (46\%), followed by foster mothers (34\%), foster fathers (9\%), and others (e.g., kinship; 11\%). The majority of youth participants were identified by caregivers as Black or African American (52\%), followed by White or Caucasian (32\%), Multiracial 10\%), and Other (6\%).

\section{Procedures}

Children and caregivers were invited to participate in the SPARK (Studying Pathways to Adjustment and Resilience in Kids) Project, a federally funded, longitudinal research project on the mechanisms of resiliency in foster youth. The state social service agency, serving as the children's legal guardian, provided consent for youth enrolled in foster care to participate. The authors' University Institutional Review Board and the State's Department of Social Services Review Board provided approval for the study. Additionally, youth and caregivers received information about the study, the voluntary nature of participation, and limits to confidentiality prior to provision of informed consent and assent at the start of each data collection meeting. Data on maltreatment history were provided by foster youth, and data on behavioral and emotional outcomes were provided by an adult caregiver (e.g., a foster parent or residential facility staff person). To be eligible for the study, youth were required to be 8 years or older, living in their current placement for at least 30 days, and have IQs of 70 or above (obtained through a screen using the Kauffman Brief Intelligence Test).

Youth and caregivers provided answers to survey questions through an audio-computer assisted self-interview (A-CASI) program on a laptop computer. The A-CASI tool is an innovative computer-based questionnaire that provides audio reading of each survey question and each answer response to participants over headphones. This method allows for maximum autonomy and confidentiality to participants, while also accommodating those who may need reading support.

Graduate research assistants with training in clinical child psychology provided a comprehensive debriefing session upon completion of each survey administration to assess for and manage any changes in mood related to participation in the project and to assess for problems related to suicidal ideation or current abuse as identified by flagged items in the ACASI program. Caregivers received and youth received gift cards for their participation. Youth and caregivers received a three-part debriefing process at the end of each data collection appointment and a follow-up phone call within 48 hours to ensure the maintenance of participant emotional well-being following completion of the survey. See XXX, XXX, XXX, and XXX (2012), for further details related to the methodology and procedures of the project.

\section{Measures}

Maltreatment-For the present study, a self-report measure on maltreatment experiences was administered that enabled youth to identify specific types of maltreatment experienced 
(e.g., Physical Abuse: "Has anyone ever kicked or punched you?"; Sexual Abuse: "Has anyone ever forced you to look at their sexual parts?"; Psychological Abuse: "Has anyone ever threatened to hurt someone very important to you?"; Neglect: "Did your parents make sure you saw a doctor if you needed one?"), the frequency of those events (e.g., "How often has someone kicked or punched you?"), severity (e.g., being exposed to sexually explicit material vs. being forced into performance of a sexual act), during their lifetime across four types of maltreatment (i.e., physical abuse, 19 items; sexual abuse, 12 items; psychological abuse, 15 items; or neglect, 22 items). Maltreatment questions were derived from the MMCS (English, 1997); the items were reworded to facilitate self-report.

Item administration followed a skip pattern such that youth received only questions about the frequency of the event if they responded in the affirmative to whether the event had occurred. For example, if a youth responded "yes" to the question, "Has any adult hit you on your head with their hand?," they would then receive a follow up question asking how many times this event occurred on a Likert scale. Items on the MMCS cover different types of maltreatment and represent a range of severity. In 2005, the LONGSCAN consortium reported that the MMCS approach provided the highest levels of measurement fidelity of maltreatment experience (English et al., 2005).

Although youth exposed to maltreatment often experience multiple types of abuse, each type of abuse (i.e., physical abuse, emotional abuse, sexual abuse, and neglect) was coded separately. To provide a comprehensive measurement model of abuse, all four types of abuse were included and accounted for in two overarching constructs of severity of abuse and frequency of abuse.

Frequency-The regularity or frequency of abuse was established through a sum of the frequency items from the maltreatment measure across maltreatment types, such that this factor represents the breadth (all types of abuse endorsed) and depth (regularity or chronicity of abuse experiences endorsed) of the youth's maltreatment.

Severity-To measure severity of maltreatment within each type of abuse, each abuse event (e.g., Mild: being slapped; Moderate: being hit with an object; Severe: being shot at with a gun) was assigned a severity level of mild, moderate, or severe abuse based on the level of danger or threat to physical integrity or emotional well-being. These levels were selected to match those severity levels represented by the MMCS as closely as possible. To obtain a final score, severity levels were weighted (i.e., mild $=1$, moderate $=2$, severe $=3$ ), and the final severity score was then divided by the number of abuse events endorsed to clean the Severity factor of variance associated with the Frequency factor. The Severity factor, therefore, included information on the type and severity of the maltreatment experiences devoid of information on the frequency and scope of those experiences.

Behavioral outcome-To measure behavioral symptoms, the Behavioral Assessment System Children 2 - Parent-report (BASC2-PRS) was used. The BASC2-PRS provides an assessment of the child's behavior at home and in the community across 150 (adolescent version for youth ages 12 years and older) - 160 (child version for youth ages $8-12$ years) items measured on a Likert scale (Reynolds \& Kamphaus, 2004). For the present study, the 
composite scales of Internalizing Problems, Externalizing Problems, and Adaptive Skills were included in the analyses. Internalizing Problems are characterized by withdrawal, low mood, or symptoms of anxiety, and the Internalizing composite consists of the anxiety, depression, and somatization subscales. Externalizing Problems are often indicated by difficulty with behavioral conduct, aggression, and behavioral restraint and the Externalizing composite includes the hyperactivity, aggression, and conduct problems subscales. The Adaptive Skills composite has five subscales: adaptability, social skills, leadership, activities of daily living, and functional communication (Reynolds \& Kamphaus, 2004). The BASC2PRS has substantial validity and reliability evidence for use as a measure of behavioral outcomes. Reliability estimates across composite indices range from alphas of .89 to .95 for parent reports of youth aged 8 and older (Reynolds \& Kamphaus, 2004).

\section{Results}

\section{Analytic Strategy}

Means, standard deviations, and Pearson correlations among the BASC2 T-scores are presented in Table 1. On subscales that are adaptive and indicative of positive behaviors, below average scores were obtained with means ranging from $40.85(S D=8.20)$ for Functional Communication to 47.31 $(S D=6.72)$ for Leadership. For subscales reflective of internalizing or externalizing problems, above average scores were obtained with means ranging from $57.06(S D=7.61)$ for attention problems to $72.91(S D=15.10)$ for conduct problems. Most scores were significantly correlated at the $p=.01$ level. Two correlations, Activities of Daily Living with Anxiety and Social Skills with Somatization, were significant at the $p=.05$ level. Missing data was minimal with covariance coverage ranging from .88 to 1.00 .

Prior to examining the effect of severity and frequency of abuse on outcomes, a confirmatory factor analysis (CFA) was conducted to establish the fit of the BASC2 indicators within three latent constructs: externalizing problem behavior, internalizing problem behavior, and adaptive behavior for the sample of youth in foster care. Because some skewness and kurtosis was observed, robust maximum likelihood estimation was used within Mplus Version 7.0 (Muthen \& Muthen, 1998-2012). The models were identified by constraining each factor variance to 1 . Because the BASC2 indicators were provided by adults, and some of the participants lived in group homes or families with multiple foster children resulting in the same adults making multiple ratings of outcomes, there was some dependency in the data. This dependency was modeled using the CLUSTER option in Mplus which adjusts the standard errors of the model parameters and the fit statistics of the model to correct for the dependency.

Four criteria were used to evaluate the fit of the CFA models: the chi-square $\left(\chi^{2}\right)$, the comparative fit index (CFI), the root-mean-square error of approximation (RMSEA), and the standardized root mean square residual (SRMR). The chi-square value is an index of the extent to which the observed variances and covariances are predicted by the system of equations specified in the model. A nonsignificant chi-square indicates that there is not a significant discrepancy between the model and the actual data. The CFI is a goodness-of-fit measure, where values above .90 or .95 indicate acceptable and excellent fit, respectively. 
The RMSEA and SRMR are measures of lack of fit, where values below .08 or .05 indicate acceptable or excellent fit, respectively. Multiple fit statistics were used because each has limitations and there is no agreed-on method for evaluating whether the lack of fit of a model is substantively important.

Initially, each factor was run in a separate model according to the manual's specifications of subscale membership and composite scores. The externalizing problem behavior model with aggression, hyperactivity, conduct problems, and adaptive behavior scales with a negative loading fit the data well, $\chi^{2}(2, N=309)=3.47, p=.18$, CFI $=0.998$, RMSEA $=0.049$, SRSMR $=0.008$. The initial model for Internalizing problem behavior with depression, somatic problems, atypical, withdrawal, attention problems, and anxiety scales as indicators did not fit the data well. Examination of the residuals for covariances indicated that there was an additional relationship between depression and anxiety that was not being modeled through the internalizing factor. Similarly there was a relationship between attention problems and atypical behavior that was not being modeled. Correlations between the residual variances of these scores were added to the model resulting in improved fit. While the chi-square value indicated a significant lack of fit, $\chi^{2}(7, \mathrm{~N}=309)=23.02, \mathrm{p}=.001$, other measures of fit less sensitive to sample size suggested that the fit was acceptable, CFI = 0.97, RMSEA $=0.086$, SRSMR $=0.032$.

The model for Adaptive Behavior with activities of daily living, leadership, functional communication, social skills, and adaptive behavior as indicators was fit similarly to the others, and once correlated, residual variances for functional communication and social skills were added the model fit the data well, $\chi^{2}(4, N=309)=6.21, p=.18, \mathrm{CFI}=0.997$, RMSEA $=0.04$, SRSMR $=0.015$. The model for just the Behavioral Symptoms Index (BSI) subscale of the BASC did not fit the data well, $\chi^{2}(15, N=473.66)=6.21, p<.001, \mathrm{CFI}=$ 0.646 , RMSEA $=0.315$, SRSMR $=0.259$. Given that the subscales included on the BSI are part of the internalizing and externalizing problem behavior scales, the BSI was dropped from further consideration in the model.

When a model was run including each of the three factors: externalizing problem behavior, internalizing problem behavior, and adaptive behavior as specified in the individual models the fit was not good, $\chi^{2}(70, N=309)=489.53, p<.001, \mathrm{CFI}=0.85, \mathrm{RMSEA}=0.14$, $\mathrm{SRSMR}=0.078$. Examination of the residuals for covariances indicated that several of subscales which fell under different latent factors were more related than the expected relationship between the latent factors could model. Therefore, relations between residual variances that were consistent with theoretical understanding of these subtests were added to the model. All associations between residual variances that were added to the model were significantly different from zero $(p<.01)$. These additions, improved the fit of the model substantially, $\chi^{2}(62, N=309)=255.82, p<.001, \mathrm{CFI}=0.93, \mathrm{RMSEA}=0.10,95 \% \mathrm{CI}$ [ $088, .114]$, SRSMR $=0.065$. Although the Chi-square value and the RMSEA indicated a lack of fit, the values for the CFI and SRSMR indicated acceptable fit for this model.

Additionally, the model fit statistics obtained from our model are consistent with or better than the fit statistics obtained by others (Kim, Kim, \& Kamphaus, 2010) who have attempted to conduct CFA on the widely-used BASC2 instrument. The model parameters for the final model can be found in Table 2. All the loadings for items on each of the three 
subscales were significantly different from zero $(p<.001)$. The standardized loadings for the items within the Externalizing Problem Behavior Factor ranged from -.56 for adaptive to . 89 for aggression. For the Internalizing Problem Behavior Factor, standardized loadings ranged from .52 to .82 . For the Adaptive Behavior Factor, standardized loadings ranged from .34 to .87 . The three behavior factors were significantly related to each other $(\mathrm{p}<.001)$ with a correlation of -.67 between Internalizing Problem Behavior and Adaptive Behavior, -.77 between Adaptive Behavior and Externalizing Problem Behavior, and .77 between Internalizing and Externalizing Problem Behavior.

Model test-Once the measurement model for youth outcomes was established, covariates were added to the model to determine what effect frequency and severity of child abuse had on child behavior outcomes. Although it could be important to establish the separate effect of severity by type (e.g., severity of sexual abuse, severity of physical abuse), given that all of the youth in the sample endorsed exposure to multiple forms of abuse, it was important to first establish how severity as a construct was related in the model.

To determine a severity score, each event endorsed by the child was apriori organized into mild, moderate, and severe categories based on the potential for each event to cause lasting harm or disability. Mild events were weighted by a 1 , moderate events by a 2 , and severe events by a 3 . The sum of those weights was divided by the sum of the total number of physical abuse, sexual abuse, and neglect events endorsed by each child. Severity of abuse had a mean of $1.62(S D=.23)$, which indicates that average participants endorsed events that were moderate to mild. Scores ranged from 1.00 for someone who endorsed only mild abuse events to 2.10 for someone who endorsed both mild and severe events.

Frequency of abuse was the sum of the frequency scores for the abuse items endorsed by each child, where $0=$ never, $1=$ almost never, $2=$ sometimes, $3=$ often, and $4=$ always. Thus, the potential score range was from 0 for someone who endorsed no abuse events to 212 for someone who indicated that all of the events occurred always. In this sample, the average frequency was $35.48(S D=23.99)$ and the highest obtained score was 137. Age was also added as a covariate in the model because participant age could be related to frequency of abuse with older participants reporting more events over their lifetimes that younger participants. The tested model included frequency and severity of abuse regressed on age, quantity and quality regressed on each of the BASC latent factors, Externalizing Problem Behavior, Internalizing Problem Behavior, and Adaptive Behavior. A correlation between frequency and severity of abuse was also modeled. The fit of the model did not change significantly with the addition of the age covariate, $\chi^{2}(98, N=309)=348.10, p<.001$, CFI $=0.92$, RMSEA $=0.09,95 \%$ CI $[.081, .101]$, SRSMR $=0.066$.

Figure 1 illustrates the relations indicated by the covariate model. There was a significant relation between age and both frequency of abuse and severity of abuse with a stronger relation between age and frequency. The results indicated a significant positive relation between severity of abuse and externalizing problem behavior and a significant negative relation between severity of abuse and adaptive behavior. Frequency of abuse did not significantly influence any of the three outcome factors. 


\section{Discussion}

The purpose of the present study was to test if commonly identified components of maltreatment have unique relations to different kinds of outcome behaviors. Although past research has often demonstrated a relation between maltreatment and outcome, the nature of maltreatment is complex, making conclusions about what aspect of the abuse event is actually predictive of outcomes perhaps less certain. The results of the present study provide some support for the notion that characteristics of the abuse matter as when tested together; severity, and not frequency of abuse was significantly related to both pathology (i.e., externalizing) and adaptive behavior. It may be important for researchers interested in further testing the maltreatment-behavioral outcome relation to consider not just if youth in their samples have an abuse history, but what kind of history and more specifically, how intense or severe the experiences were.

The results are consistent with other efforts in the field to isolate and identify elements of maltreatment experiences and understand their unique functions in youth well-being (English et al., 2005). Moreover, the results are quite similar to the findings of Litrownik et al. (2005) where severity of abuse (with type included) was predictive of externalizing and adaptive behavior in youth exposed to maltreatment. The results are also consistent with the cluster analysis by Higgins (2004) in which behavioral outcome was best predicted by maltreatment when maltreatment was clustered according to low, medium, and high degrees of abuse. Higgins concluded that rather than separate abuse by type, when maltreatment was organized by the frequency and severity of abuse combined, the result was meaningful differences in behavioral outcome. The results from Manly et al. (1994) are also somewhat consistent with the present findings. That is, Manly and colleagues found that severity and frequency were predictive of child symptoms; however, frequency was measured as the number of reports to state authorities perhaps explaining the different result for the present study. Overall, the present findings add to the field by replicating the findings of Litrownik et al. and others and extend the knowledge base by providing evidence that when compared to and separated from severity of abuse, frequency of abuse may have little relation to behavioral outcomes.

Beyond the important findings related to operationalization of maltreatment, this study also provides further evidence for relations between maltreatment and outcomes. Given the rather long list researchers have developed of possible negative outcomes for youth exposed to maltreatment, the present study adds to the field by including not only variables measuring pathology, but also adaptive behavior. Not all youth exposed to maltreatment demonstrate the same outcome and it is possible that given the range of severity and frequency of their experiences, not all youth were necessarily likely to demonstrate significant maladjustment.

Although the results supported the study predictions, the results for internalizing behavior were unexpected. It is not the case that abuse experiences are more likely to be related to externalizing behavior only, so it was reasonable, and in the name of being comprehensive, to include internalizing behavior in the model test. It is possible that because behavioral outcome was measured via caregiver-report, the experiences of internalizing behavior were 
less likely to be identified as other research suggests youth self-report to be the best method for assessing internalizing symptoms (van de Looij-Jansen, Jansen, de Wilde, Erik, Donker, \& Verhulst, 2011). It is also possible that given the modifications made to improve the fit of the measurement model for the BASC2, the makeup of the internalizing scale in the present sample was not entirely consistent with the manual, resulting in a construct was not captured in a way uniform with other studies that use traditional methods to calculate scores.

Considered another way, however, the present study adds some rigor to the typical approach of measuring behavioral outcome in foster youth by including a measurement model of the behavioral outcome measure prior to testing relations between the variables of interest. Although this step is rarely taken, it is important. The present results suggest that items that makeup standard measures of adjustment may not accurately reflect the structure of how behavioral constructs operate in a special population such as youth in foster care.

Although it would be inaccurate to suggest that the present results indicate that the number of times something abusive happens does not impact a child's well-being, perhaps it could be suggested that when severity is also considered, the frequency of events may be less important in predicting how a child will adjust. It is possible that the measurement of frequency was inadequate in that youth may not be good reporters of how often a given event occurred. Moreover, it is possible that when maltreatment is measured as present or absent or is focused on a specific type only, findings, although often compelling, may be misleading because this approach ignores the rather qualitatively different elements of the maltreatment experience (Ahmadkhaniha et al., 2007).

The present study is not the first effort by the field to untangle the relevant components of abuse and examine their unique impacts (Portwood, 1999), but it is a part of a growing body of literature that suggests that researchers may want to be careful not to assume that any sample of youth exposed to maltreatment, even samples restricted to a given type of abuse, are somehow homogenous in regard to the nature of their experiences. This potential difference in the level, amount, or degree of abuse (even across types) may be important when examining how maltreatment is related to other youth outcomes.

\section{Maltreatment as a predictor}

Frequency of abuse is an important consideration for youth in foster care. For some types of abuse, especially neglect, it is often the reoccurrence and not the single incidence of failure to provide for a child's basic needs that is of concern to social welfare agencies making home removal decisions. Most research on frequency, however, when not confounded with chronicity, examines how rates of abuse rise or fall in various settings (e.g., emergency rooms, among foster placements), and little research is actually devoted to testing the predictive value of repeated instances of abuse (Benedict, Zuravin, Brandt, \& Abbey, 1994) on behavioral outcome for youth. Perhaps this is understandable given that severity and frequency are often intertwined, meaning that some kinds of abuse, if deemed severe, only have to happen one time (i.e., sexual intercourse) for the abuse to be considered a significant concern. In most studies of sexual abuse, for example, the rate of abuse or number of times one has been abused is not often the focus of the study, which makes it difficult to know 
what role severity and frequency play in the commonly found maladjustment among child sexual abuse victims (Tyler, 2002).

The present study also adds to the field by providing an assessment of maltreatment based solely on youth-report. Although it is possible that a child's memory for events could add error to the data, the use of case file data also has problems and likely would not include reports of abuse that the child may recall, but do not rise to the level of evidence for state authorities to act. Because of the complexity of the maltreatment construct, the researchers in the field of child maltreatment as a whole may struggle with a somewhat disconnected collection of evidence for the maltreatment-maladjustment relation. When maltreatment is found to relate to maladjustment, the most common method for identifying this relation is via a maltreatment present or absent measurement method. Given the heterogeneity of abuse experiences, it is hoped that results from studies such as the present study can assist the field in being clear about what it is about the abuse that is predictive of outcomes. Moreover, failure to account for varying levels of exposure could help explain the long list of possible reactions to abuse. That is, by combining youth exposed to maltreatment into one group, it is possible that researchers will find that any and all behaviors are related to abuse with little ability to know if there is an element of abuse, such as severity, that perhaps is really driving the association with outcome. It is also possible that knowing the relevant elements of abuse could be prescriptive for interventions, where the focus could be drawn on the primary mechanisms responsible for behavioral outcome differences among victims.

The present study included type of abuse within the overarching framework of the frequency and severity constructs. All of the youth in the sample were known to have had exposure to more than one type of abuse at the level that required removal from their homes. That is, abuse type was combined making the frequency and severity scores averages across all abuse types. In this way, the findings from the present study could be considered as the "dose" of abuse exposure across types. The present conceptualization was essentially the answer to the question, "What have you experienced, how often and how bad?" In a sense, type of abuse was not separated from frequency and severity, but frequency and severity were separated analytically from each other to meet the study aims of determining their possible unique contributions. The present results do not argue against other studies that might continue to group for example, youth with a physical abuse history together and examine outcomes, but it does suggest that such an endeavor should likely consider assessing for more than just if one was every physically abused and include some review of the severity of the abuse to better understand the possible relation to behavioral outcome.

\section{Limitations and directions for future research}

The findings for the present study provide important information for how researchers and the field may want to consider the construct of child maltreatment and its relation to behavioral outcome. In addition to providing evidence for the differing roles of the components of abuse and including a comprehensive approach to behavioral outcome by including adaptive behavior, the study is not without limitations. One, the data is crosssectional, so causal connections between the variables of interest are not indicated by the current findings. It would be important for future studies to follow youth over time to 
determine if the components of their maltreatment experiences maintain the same relations found here or if these relations are sensitive to change over time.

Two, the sample was comprised entirely of youth in foster care and may not generalize to youth exposed to maltreatment who have not been identified to state authorities. It is perhaps expected that youth in foster care have experienced either more frequent or severe instances of abuse than youth in the general population, which in some ways may have made the relations supported here easier to identify than youth whose exposure to maltreatment is fairly infrequent or mild in nature.

Three, the present study included caregiver report, either foster parent or residential staff member who knew the child for a reasonable amount of time, but the design did not control for how long the youth had been in care or the type of setting (i.e., residential care vs. traditional home-based care). Although it is possible that the sample of youth in foster care was somewhat heterogeneous in regard to placement history and length of time in care, the focus of the present study was on events since placement or abuse history before coming into care. As alternative care was not the emphasis of the present study, but could be an important factor, additional research is needed to determine how placement history might impact behavioral ratings by caregivers for youth in foster care. Moreover, whether one is placed in a traditional home or a residential facility is likely the result of many factors, not the least of which may be availability of placement options. Because where one is placed at a given time is variable and subject to change, additional research is also needed to determine if type of placement and length of placement impacts the abuse-behavioral outcome relation.

Four, it is likely that the significant path for severity of abuse to behavioral outcome could be confounded by the age of the child at the time of the abuse. That is, some events could be considered severe depending on the age of the child (i.e., leaving a child at home alone). Although age was considered in the present study as a covariate given the wide age range of the sample, it would be important for future research to consider how age at the time of abuse may be accounted for in future model tests of the abuse-outcome relation. It is also important to note that the designations for severity (i.e., mild, moderate, severe) were determined apriori based on harm or potential for lasting injury or disability and not based on the child's report of one event being worse than another. Although severity was weighted within type (i.e., hierarchy of severity within physical abuse separate from hierarchy within sexual abuse), it is possible that youth may not view, for example, being slapped as less severe than being burned. It would be important for future research to also consider youth self-report not just to document if events occurred, but also the youth's interpretation of events as a possible moderator of the exposure-adjustment relation.

Finally, the results of the present study add to the field by documenting the unique relation severity and frequency of abuse do and do not have with various behavioral outcomes. It would be important for future research to consider that abuse does not occur in a vacuum and that when present, abuse experiences are often confounded by other risk and stress event that in some cases, pre-date the abuse exposure (Dube et al., 2001) and are also related to the child's overall well-being and adjustment. For example, the present sample was composed 
of youth in foster care, but did not assess length of time in care or the nature of the child's foster care experience, which may also impact ratings of behavioral health by caregivers. Exposure to abuse and placement in foster care are important, often life changing experiences for youth and research that attempts to investigate the relations between life experiences and outcome is challenging. The results of the present study provide some evidence that close attention to the nature of the abuse exposure at least provides some meaningful information on how the field might understand the plethora of poor adjustment outcomes often documented by other studies. The path to clarity about child abuse is likely a long one, but it is hoped that the results of the present study provide the field with important considerations for the conceptualization of abuse and behavioral outcome for youth in foster care.

\section{Acknowledgments}

The current project was made possible by funding from the National Institutes of Mental Health, RO1 grant MH079252-03.

\section{References}

Afifi TO, McMillan KA, Asmundson GJ, Pietrzak RH, Sareen J. An examination of the relation between conduct disorder, childhood and adulthood traumatic events, and posttraumatic stress disorder in a nationally representative sample. Journal of Psychiatric Research. 2011; 45:15641572.10.1016/j.jpsychires.2011.08.005 [PubMed: 21903224]

Ahmadkhaniha HR, Shariat SV, Torkaman-Nejad S, Moghadam MMH. The frequency of sexual abuse and depression in a sample of street children of one of deprived districts of Tehran. Journal of Child Sexual Abuse. 2007; 16(4):23-35. http://dx.doi.org/10.1300/J070v16n04_02. [PubMed: 18032244]

Arata CM, Langhinrichsen-Rohling J, Bowers D, O'Farrill-Swails L. Single versus multi-type maltreatment. Journal of Aggression, Maltreatment \& Trauma. 2005; 11(4):29-52.10.1300/ J146v11n04_02

Barnett D, Manly JT, Cicchetti D. Defining child maltreatment: The interface between policy and research. Child abuse, child development, and social policy. 1993; 8:7-73.

Benedict MI, Zuravin S, Brandt D, Abbey H. Types and frequency of child maltreatment by family foster care providers in an urban population. Child Abuse \& Neglect. 1994; 18:577-585. http:// dx.doi.org/10.1016/0145-2134(94)90084-1. [PubMed: 7922732]

Bolger KE, Patterson CJ. Developmental pathways from child maltreatment to peer rejection. Child Development. 2001; 72:549-568. http://dx.doi.org/10.1111/1467-8624.00296. [PubMed: 11333084]

Bryant SL, Range LM. Type and severity of child abuse and college students' lifetime suicidality. Child Abuse \& Neglect. 1997; 21:1169-1176. http://dx.doi.org/10.1016/S0145-2134(97)00092-6. [PubMed: 9429769]

Currie J, Spatz Widom C. Long-term consequences of child abuse and neglect on adult economic wellbeing. Child Maltreatment. 2010; 15:111-120.10.1177/1077559509355316 [PubMed: 20425881]

DePanfilis D, Zuravin SJ. Rates, patterns, and frequency of child maltreatment recurrences among families known to CPS. Child Maltreatment. 1998; 3:27-42.10.1177/1077559598003001003

Dube SR, Anda RF, Felitti VJ, Croft JB, Edwards VJ, Giles WH. Growing up with parental alcohol abuse: Exposure to childhood abuse, neglect, and household dysfunction. Child Abuse \& Neglect. 2001; 25:1627-1640. http://dx.doi.org/10.1016/S0145-2134(01)00293-9. [PubMed: 11814159]

English DJ, Upadhyaya MP, Litrownik AJ, Marshall JM, Runyan DK, Graham JC, Dubowitz H. Maltreatment's wake: The relationship of maltreatment dimensions to child outcomes. Child Abuse \& Neglect. 2005; 29:597-619. http://dx.doi.org/10.1016/j.chiabu.2004.12.008. [PubMed: 15970327] 
Éthier LS, Lemelin JP, Lacharité C. A longitudinal study of the effects of chronic maltreatment on children's behavioral and emotional problems. Child Abuse \& Neglect. 2004; 28:1265-1278. http://dx.doi.org/10.1016/j.chiabu.2004.07.006. [PubMed: 15607769]

Fantuzzo JW, Perlman SM, Dobbins EK. Types and timing of child maltreatment and early school success: A population-based investigation. Children and Youth Services Review. 2011; 33:14041411. http://dx.doi.org/10.1016/j.childyouth.2011.04.010.

Fassler IR, Amodeo M, Griffin ML, Clay CM, Ellis MA. Predicting long-term outcomes for women sexually abused in childhood: Contribution of abuse severity versus family environment. Child Abuse \& Neglect. 2005; 29:269-284. http://dx.doi.org/10.1016/j.chiabu.2004.12.006. [PubMed: 15820543]

Hahm HC, Lee Y, Ozonoff A, Van Wert MJ. The impact of multiple types of child maltreatment on subsequent risk behaviors among women during the transition from adolescence to young adulthood. Journal of Youth and Adolescence. 2010; 39:528-540. http://dx.doi.org/10.1007/ s10964-009-9490-0. [PubMed: 20020190]

Herrenkohl RC, Herrenkohl TI. Assessing a child's experience of multiple maltreatment types: Some unfinished business. Journal of Family Violence. 2009; 24:485-496. http://dx.doi.org/10.1007/ s10896-009-9247-2. [PubMed: 20161642]

Higgins DJ, McCabe MP. Multiple forms of child abuse and neglect: Adult retrospective reports. Aggression and Violent Behavior. 2001; 6:547-578. http://dx.doi.org/10.1016/ S1359-1789(00)00030-6.

Higgins DJ, McCabe MP. Maltreatment and family dysfunction in childhood and the subsequent adjustment of children and adults. Journal of Family Violence. 2003; 18:107-120. http:// dx.doi.org/10.1023/A:1022841215113.

Jaffee SR, Caspi A, Moffitt TE, Polo-Tomas M, Taylor A. Individual, family, and neighborhood factors distinguish resilient from non-resilient maltreated children: A cumulative stressors model. Child Abuse \& Neglect. 2007; 31:231. http://dx.doi.org/10.1016/j.chiabu.2006.03.011. [PubMed: 17395260]

Keiley MK, Howe T, Dodge K, Bates J, Pettit G. Timing of abuse: Group differences and developmental trajectories. Development and Psychopathology. 2001; 13:891-912. Retrieved from http://search.proquest.com/docview/619647941 ?accountid=14556. [PubMed: 11771913]

Kim S, Kamphaus RW. Is aggression the same for boys and girls? Assessing measurement invariance with confirmatory factor analysis and item response theory. School Psychology Quarterly. 2010; 25:45-61.

Lau AS, Leeb RT, English D, Graham JC, Briggs EC, Brody KE, Marshall JM. What's in a name? A comparison of methods for classifying predominant type of maltreatment. Child Abuse \& Neglect. 2005; 29:533-551.10.1016/j.chiabu.2003.05.005 [PubMed: 15970324]

Lau AS, Weisz JR. Reported maltreatment among clinic-referred children: Implications for presenting problems, treatment attrition, and long-term outcomes. Journal of the American Academy of Child \& Adolescent Psychiatry. 2003; 42:1327-1334. http://dx.doi.org/10.1097/01.CHI. 0000085754.71002.14. [PubMed: 14566170]

Leeb, RT.; Paluzzi, L.; Melanson, C.; Simon, T.; Arias, I. Child maltreatment surveillance: Uniform definitions for public health and recommended data elements (Version 1.0.). Atlanta, GA: Centers for Disease Control and Prevention, National Center for Injury Prevention and Control; 2008.

Litrownik AJ, Lau A, English DJ, Briggs E, Newton RR, Romney S, Dubowitz H. Measuring the severity of child maltreatment. Child Abuse \& Neglect. 2005; 29:553-573. http://dx.doi.org/ 10.1016/j.chiabu.2003.08.010. [PubMed: 15970325]

Lobbestael J, Arntz A, Bernstein DP. Disentangling the relationship between different types of childhood maltreatment and personality disorders. Journal of Personality Disorders. 2010; 24:285295. http://dx.doi.org/10.1521/pedi.2010.24.3.285. [PubMed: 20545495]

Loftus EF. Planting misinformation in the human mind: A 30-year investigation into the malleability of memory. Learning and Memory. 2005; 12:361-366.10.1101/lm94705 [PubMed: 16027179]

Maniglio R. Child sexual abuse in the etiology of depression: A systematic review of reviews. Depression and Anxiety. 2010; 27:631-642. http://dx.doi.org/10.1002/da.20687. [PubMed: 20336807] 
Manly JT, Cicchetti D, Barnett D. The impact of subtype, frequency, chronicity, and severity of child maltreatment on social competence and behavior problems. Development and Psychopathology. 1994; 6:121-143.10.1017/S0954579400005915

McGee RA, Wolfe DA, Yuen SA, Wilson SK, Carnochan J. The measurement of maltreatment: A comparison of approaches. Child Abuse \& Neglect. 1995; 19:233-249. http://dx.doi.org/ 10.1016/0145-2134(94)00119-F. [PubMed: 7780784]

Miller GE, Chen E, Parker KJ. Psychological stress in childhood and susceptibility to the chronic diseases of aging: moving toward a model of behavioral and biological mechanisms. Psychological bulletin. 2011; 137:959. http://dx.doi.org/10.1037/a0024768. [PubMed: 21787044]

Mills R, Alati R, O'Callaghan M, Najman JM, Williams GM, Bor W, Strathearn L. Child abuse and neglect and cognitive functioning at 14 years of age: Findings from a birth cohort. Pediatrics. 2011; 127:4-10.10.1542/peds.2009-3479 [PubMed: 21135010]

Pears KC, Kim HK, Fisher PA. Psychosocial and cognitive functioning of children with specific profiles of maltreatment. Child Abuse \& Neglect. 2008; 32:958. http://dx.doi.org/10.1016/j.chiabu. 2007.12.009. [PubMed: 18995901]

Portwood SG. Coming to terms with a consensual definition of child maltreatment. Child Maltreatment. 1999; 4:56-68.10.1177/1077559599004001006

Runyan DK, Litrownik AJ. Introduction to special issue: LONGSCAN and family violence. Journal of Family Violence. 2003; 18:1-4. http://dx.doi.org/10.1023/A:1021468429435.

Rutter ML. Nature-nurture integration: The example of antisocial behavior. American Psychologist. 1997; 52:390. http://dx.doi.org/10.1037/0003-066X.52.4.390.

Slep AMS, Heyman RE. Creating and field-testing child maltreatment definitions: Improving the reliability of substantiation determinations. Child Maltreatment. 2006; 11:217-236. http:// dx.doi.org/10.1177/1077559506288878. [PubMed: 16816320]

Tanaka, M.; Wekerle, C.; Schmuck, ML.; Paglia-Boak, A. The linkages among childhood maltreatment, adolescent mental health, and self-compassion in child welfare adolescents. Child Abuse \& Neglect. 2011. http://dx.doi.org/10.1016/j.chiabu.2011.07.003

Thompson R, Tabone JK. The impact of early alleged maltreatment on behavioral trajectories. Child Abuse \& Neglect. 2010; 34:907-916. http://dx.doi.org/10.1016/j.chiabu.2010.06.006. [PubMed: 21111247]

Trocmé NM, Tourigny M, MacLaurin B, Fallon B. Major findings from the Canadian incidence study of reported child abuse and neglect. Child Abuse \& Neglect. 2003; 27:1427-1439. http:// dx.doi.org/10.1016/j.chiabu.2003.07.003. [PubMed: 14644059]

Tyler KA. Social and emotional outcomes of childhood sexual abuse. A review of recent research. Aggression\& Violent Behavior. 2002; 7:567-589. http://dx.doi.org/10.1016/ S1359-1789(01)00047-7.

Van de Looij-Jansen PM, Jansen W, de Wilde EJ, Donker M, Verhulst FC. Discrepancies between parent-child reports of internalizing problems among preadolescent children: Relationships with gender, ethnic background, and future internalizing problems. Journal of Early Adolescence. 2011; 31:443-462. http://dx.doi.org/10.1177/0272431610366243. 


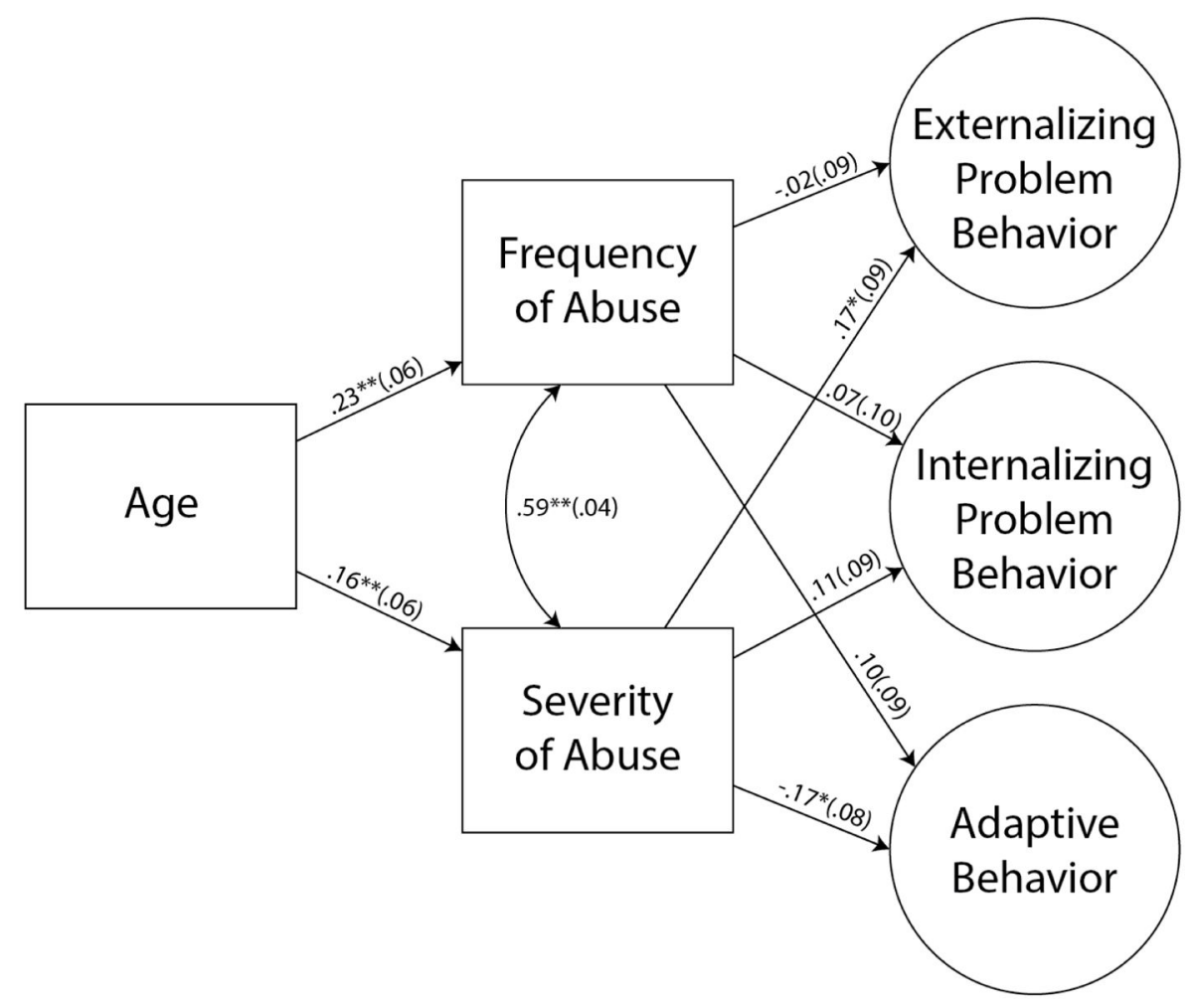

Figure 1.

Covariate model of age, frequency of abuse, severity of abuse, and BASC2 Outcomes (Externalizing Behavior, Internalizing Behavior, and Adaptive Behavior). 


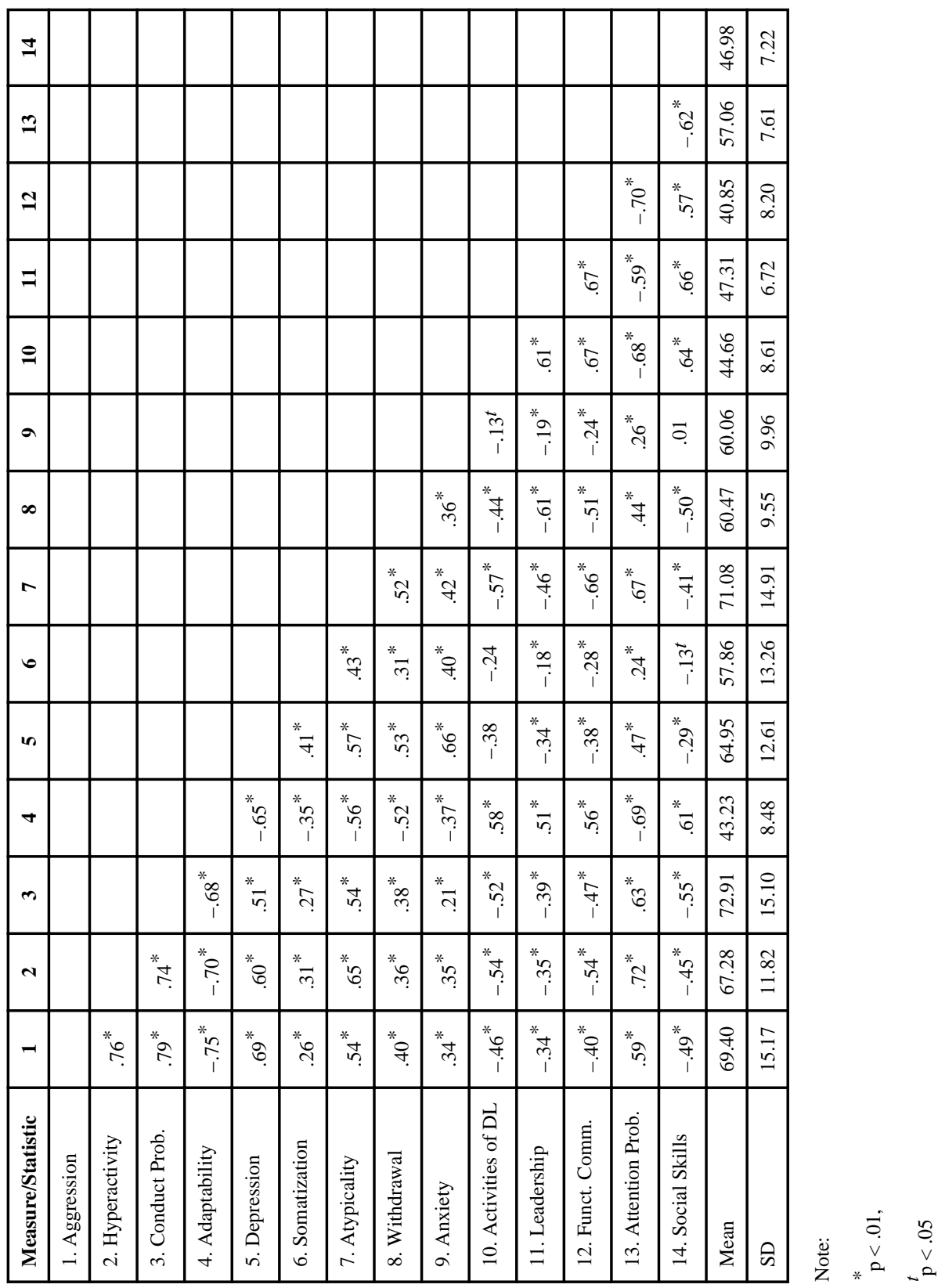




\section{Table 2}

Item Factor Loadings for the BASC2

\begin{tabular}{|c|c|c|}
\hline Item Factor Loadings & Estimate & SE \\
\hline \multicolumn{3}{|l|}{ Externalizing Behavior } \\
\hline Aggression & .89 & .02 \\
\hline Hyperactivity & .86 & .02 \\
\hline Conduct Problems & .85 & .02 \\
\hline Adaptability & -.56 & .07 \\
\hline \multicolumn{3}{|l|}{ Internalizing Behavior } \\
\hline Depression & .82 & .03 \\
\hline Somatization & .52 & .06 \\
\hline Atypical & .56 & .06 \\
\hline Withdrawn & .68 & .04 \\
\hline Anxiety & .55 & .06 \\
\hline \multicolumn{3}{|l|}{ Adaptive Behavior } \\
\hline Activities of Daily Living & .79 & .03 \\
\hline Leadership & .69 & .04 \\
\hline Functional Communication & .82 & .03 \\
\hline Adaptability & .34 & .08 \\
\hline Attention Problems & -.87 & .02 \\
\hline Atypical & -.34 & .06 \\
\hline Social Skills & .72 & .04 \\
\hline \multicolumn{3}{|l|}{ Correlated Residual Variances } \\
\hline Depression with Anxiety & .38 & .07 \\
\hline Depression with Aggression & .43 & .08 \\
\hline Functional Comm. with Soc. Skills & -.17 & .07 \\
\hline Functional Comm. with Aggr. & .26 & .08 \\
\hline Leadership with Withdrawn & -.52 & .05 \\
\hline Leadership with Social Skills & .32 & .06 \\
\hline Social Skills with Anxiety & .27 & .05 \\
\hline Atten. Prob. with Hyperactivity & .38 & .07 \\
\hline Atypical with Adaptability & .35 & .08 \\
\hline Withdrawn with Social Skills & -.38 & .06 \\
\hline \multicolumn{3}{|l|}{ Residual Variances } \\
\hline Hyperactivity & .26 & .04 \\
\hline Aggression & .21 & .03 \\
\hline Conduct Problems & .29 & .04 \\
\hline Anxiety & .70 & .06 \\
\hline Depression & .34 & .05 \\
\hline Somatization & .73 & .06 \\
\hline
\end{tabular}




\begin{tabular}{|l|c|c|}
\hline Item Factor Loadings & Estimate & SE \\
\hline Adaptability & .28 & .03 \\
\hline Social Skills & .48 & .05 \\
\hline Leadership & .52 & .05 \\
\hline Activities of Daily Living & .38 & .05 \\
\hline Functional Communication & .33 & .04 \\
\hline Atypical & .31 & .04 \\
\hline Withdrawn & .54 & .06 \\
\hline Attention Problems & .25 & .03 \\
\hline
\end{tabular}

